\title{
The Use of VTE prophylaxis in relatioN to patiEnt risk profiling (TUNE-IN) Wave 2 study
}

\author{
B F Jacobson, ${ }^{1}$ MB ChB, FRCS (Glasg), MMed (Haem), FCPath (SA), PhD (Med); S Louw, ${ }^{1}$ MB BCh, FCPath (Haem), MMed (Haem); \\ W J Riback, ${ }^{2} \mathrm{MB}$ BCh, DA (CMSA) \\ ${ }^{1}$ Department of Molecular Medicine and Haematology, Faculty of Health Sciences, University of the Witwatersrand and \\ National Health Laboratory Service, Johannesburg, South Africa \\ 2 Sanofi, South Africa
}

Corresponding author: S Louw (susan.louw@nhls.ac.za)

\begin{abstract}
Background. The TUNE-IN (The Use of VTE prophylaxis in relatioN to patiEnt risk profiling) study evaluated venous thromboembolism (VTE) risk assessment and prophylaxis in private medical and surgical inpatients in Gauteng Province, South Africa. The study concluded that of the 608 patients enrolled, 54.1\% were clinically evaluated to be at risk for VTE. A VTE risk assessment model (RAM), the Caprini score, increased the rate to $74.6 \%$.

Objectives. TUNE-IN Wave 2, an extension of TUNE-IN, was conducted on a national level including the public sector, focusing on surgical inpatients.

Methods. The study was a national, prospective, non-interventional, multisite, epidemiological disease registry enrolling 453 surgical inpatients. The perceived clinical VTE risk, VTE risk score on Caprini RAM, VTE prophylaxis and clinical details were documented during a baseline visit. A bleeding risk score was provided.

Results. Of the cohort, 269 patients (59.4\%) were assessed to be at risk for VTE before applying the RAM. All patients (100\%), however, were at risk on the RAM score. Early mobilisation and assessment of the VTE risk as low were the most frequent reasons for non-prescription of prophylaxis. Only 15 patients in the private and 2 in the public sector were assessed as having a bleeding risk. Chemoprophylaxis differed between the healthcare sectors, with low-molecular-weight heparin predominating in the private sector and unfractionated heparin being prescribed only in the public sector.

Conclusion. VTE risk assessment and prophylaxis need to improve in both the public and the private sectors. A formal RAM will improve identification of patients at risk of VTE.

S Afr Med J 2014;104(12):880-884. DOI:10.7196/SAMJ.8456
\end{abstract}

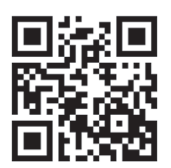

The ENDORSE (Epidemiologic International Day for the Evaluation of Patients at Risk for Venous Thromboembolism in the Acute Hospital Care Setting) study ${ }^{[1]}$ demonstrated that, globally, more than $50 \%$ of hospitalised patients are at risk of venous thromboembolism (VTE). However, adequate prophylaxis, as defined by the 7th American College of Chest Physicians (ACCP) guidelines, ${ }^{[2]}$ was given to only approximately $58 \%$ of surgical patients and $40 \%$ of medical patients. The proportion of patients likely to benefit from receiving adequate VTE prophylaxis now needs to be determined.

The TUNE-IN (The Use of VTE prophylaxis in relatioN to patiEnt risk profiling) study ${ }^{[3]}$ was conducted to evaluate common practice in the assessment of VTE risk and concurrent prescription of prophylaxis in the private inpatient healthcare setting in Gauteng Province, South Africa (SA). The study documented the perceived clinical VTE risk, actual VTE risk score according to the modified Caprini risk assessment model (RAM) (Table 1), VTE prophylaxis 


\section{Table 1. Modified Caprini risk model}

Each of the following risk factors represents 1 point:

Age 41 - 59 years

Minor surgery planned

History of previous major surgery ( $<1$ month)

Varicose veins

History of inflammatory bowel disease

Swollen legs (current)

Obesity $\left(\mathrm{BMI}>30 \mathrm{~kg} / \mathrm{m}^{2}\right)$

Acute myocardial infarction ( $<1$ month)

Congestive heart failure $(<1$ month)

Sepsis ( $<1$ month)

Serious lung disease incl. pneumonia ( $<1$ month)

Abnormal pulmonary function (COPD)

Medical patient currently on bed rest

Leg plaster cast or brace

Central venous access

Each of the following risk factors represents 2 points:

Age 60 - 74 years

Major surgery ( $>60$ minutes)

Arthroscopic surgery ( $>60$ minutes)

Laparoscopic surgery (>60 minutes)

Previous malignancy

Morbid obesity (BMI $>40 \mathrm{~kg} / \mathrm{m}^{2}$ )

Each of the following risk factors represents 3 points:

Age $\geq 75$ years

Major surgery lasting 2 - 3 hours

BMI $>50 \mathrm{~kg} / \mathrm{m}^{2}$ (venous stasis syndrome)

History of SVT, DVT/PE

Family history of DVT/PE

Present cancer or chemotherapy

Congenital thrombophilia

Positive factor V Leiden

Positive prothrombin 20210A

Elevated serum homocysteine

Acquired thrombophilia

Positive lupus anticoagulant

Elevated anticardiolipin antibodies

HIT

Other thrombophilia

Each of the following risk factors represents 5 points:

Elective major lower extremity arthroplasty

Hip, pelvis or leg fracture ( $<1$ month)

Stroke ( $<1$ month)

Multiple trauma $(<1$ month)

Acute spinal cord injury (paralysis) ( $<1$ month)

Major surgery $>3$ hours
Table 1. (continued) Modified Caprini risk model

For women only (each risk factor represents 1 point):

Oral contraceptives or HRT

Pregnancy or postpartum (<1 month)

History of unexplained stillborn infant, recurrent spontaneous

abortion $(\geq 3)$, premature birth with toxaemia or growth-restricted infant

$\mathrm{BMI}=$ body mass index; $\mathrm{COPD}=$ chronic obstructive pulmonary disease; SVT $=$ superficial venous thrombosis; DVT/PE = deep-vein thrombosis/pulmonary embolism; HIT = heparininduced thrombocytopenia; HRT = hormone replacement therapy.

prescribed and mobilisation at varying post-discharge dates. The TUNE-IN study was published in the SAMJ in February $2012,{ }^{[4]}$ with the following findings:

- Of the patients, $54.1 \%$ were considered to be at risk for VTE on clinical assessment.

- Concurrent use of a VTE RAM increased this figure to 74.6\%.

- Adequate prophylaxis, as per VTE prophylaxis guidelines, was only administered to $67.9 \%$ of surgical and $70.9 \%$ of medical patients who were at risk of VTE.

The TUNE-IN Wave 2 study was an extension of the original TUNEIN to a national level and included public sector hospitals to assess whether the results of the original study were reflective of both healthcare sectors across SA. In addition, TUNE-IN Wave 2 focused exclusively on surgical patients and data were only collected once at baseline, with no subsequent mobilisation assessment.

\section{Methods}

\section{Settings and patients}

The TUNE-IN Wave 2 study enrolled 453 patients in a national, prospective, non-interventional, multisite, epidemiological disease registry over the period September 2009 - October 2010. The patients were surgical inpatients at 18 sites in private and public sector hospitals across the country. The sites were randomly selected.

\section{Data collection}

The perceived clinical VTE risk, VTE risk score according to the modified Caprini RAM (Table 1), VTE prophylaxis prescribed and surgical subspecialty were documented during a baseline visit.

\section{VTE risk scoring}

Treating doctors (surgeons, gynaecologists and anaesthetists) at participating sites were asked to evaluate VTE risk clinically, i.e. without using any official scoring system. The patients were then re-evaluated via an approved RAM (Table 1). This scoring system assigns various risk factors a specific value. The total VTE risk is then calculated by adding the values and assigns the patient to a risk category, i.e. low, moderate, high and highest (Table 2). The decision regarding administration of VTE prophylaxis was left to the treating doctor. A bleeding risk reminder was also provided (Table 3). Consecutive patients meeting the entry criteria were enrolled. A baseline VTE risk assessment was undertaken and VTE prophylaxis documented. No risk factor score for HIV disease was given; however, as it poses a significant risk for VTE, it was noted as an additional comment wherever relevant.

\section{Patient characteristics}

TUNE-IN Wave 2 enrolled 453 surgical inpatients within the allocated time period; this was deemed adequate for statistical analysis. The majority of the patients (69.5\%) were from the private sector, with 
$30.5 \%$ from the public sector. The inclusion criteria for the study were as follows: surgical inpatient, $\geq 18$ years old, with signed patient informed consent and data release consent form available. Patients not meeting the inclusion criteria, those currently on anticoagulation therapy, e.g. warfarin, and pregnant women were excluded.

The mean age of patients in the private sector was 50.1 years v. 45.4 in the public sector. Interestingly, the patients in the private sector had higher weights $(77.9 \mathrm{~kg}$ v. 70.0 $\mathrm{kg}$ ) and body mass indices than the public sector patients. The majority of the patients were female, with a female/male ratio of 69:31. Just over half (50.2\%) were classified as being general surgery patients and $21.8 \%$ as gynaecological patients. The remainder of the patients were distributed across various surgical subspecialties including orthopaedics (8.0\%), vascular surgery (2.5\%) and urology (2.2\%). A significant proportion (13.3\%) of primarily surgical patients had concomitant medical comorbidities.

Table 2. Risk levels and recommendation according to risk level

\begin{tabular}{llll}
\hline Total risk factor score & Incidence of DVT & Risk level & Prophylaxis regimen \\
\hline $0-1$ & $<10 \%$ & Low & $\begin{array}{l}\text { No specific measures, early } \\
\text { ambulation }\end{array}$ \\
2 & $10-20 \%$ & Moderate & ES/GCS, IPC, LDUH or LMWH \\
$3-4$ & $20-40 \%$ & High & IPC, LDUH or LMWH \\
$\geq 5$ & $40-60 \%$ & Highest & $\begin{array}{l}\text { Pharmacological LDUH, LMWH, } \\
\text { warfarin or F-Xa inhibitor alone } \\
\text { or in combination with ES/GCS } \\
\end{array}$ \\
& & & or IPC
\end{tabular}

ES/GCS = elastic stockings/graduated compression stockings; IPC = intermittent pneumatic compression; $\mathrm{LDUH}=$ low-dose unfractionated heparin; LMWH = low-molecular-weight heparin; F-Xa = factor Xa.

Table 3. Bleeding risk assessment

Has the patient, or a blood relative, ever received medical care for a bleeding tendency, e.g.:

Nose bleeds (epistaxis)

Excessive bleeding after a dental procedure or extraction

Excessive bleeding after trauma or surgery

Excessive bleeding after tonsillectomy

Excessive bleeding during childbirth or during menstruation

Excessive bleeding from minor cuts?

Does the patient bruise easily?

Does the patient have a history of heparin-induced thrombocytopenia?

Is the patient's platelet count $<100 \times 10^{9} / \mathrm{L}$ or $>1000 \times 10^{9} / \mathrm{L}$ ?

Is the patient taking aspirin or clopidogrel?

Does the patient have kidney or liver disease?

Is the patient on any medication (such as NSAIDs, and including natural/homeopathic medication, e.g. garlic tablets, arnica, Procydin) that increases the risk of bleeding?

NSAIDs $=$ non-steroidal anti-inflammatory drugs.

\section{Statistical analysis}

The following were key measurements at the once-off baseline assessment: (i) clinical VTE risk assessment; (ii) VTE risk assessment with the Caprini RAM; (iii) surgical subspecialty; and (iv) type, duration and dosage of VTE prophylaxis prescribed.

\section{Results and discussion}

Before applying the Caprini VTE risk factor assessment scale, the treating doctors deemed 269 patients $(59.4 \%)$ to be at risk for VTE (private $n=149(47.3 \%)$, public $n=120$ $(87.0 \%))$ and $182(40.2 \%)$ not to be at risk (private $n=164$ (52.1\%), public $n=18(13.0 \%)$ ) (Table 4 and Fig. 1). VTE risk assessments were not recorded for two private patients. The 269 patients clinically assessed as being at risk for VTE were assigned the following risk levels: 'low' risk $n=3$, 'moderate' risk $n=18$, 'high' risk $n=88$, and 'highest' risk $n=160$. However, all the patients included in the study were at risk to a greater or lesser extent as assessed on the Caprini RAM (Table 4 and Fig. 1). Applying the RAM resulted in an additional 184 patients being classified as at risk for VTE.

\section{Reasons for not prescribing prophylaxis}

Why prophylaxis was not prescribed was reported for 91 patients (private $n=63$, public $n=28$ ), early mobilisation and low VTE risk being the most frequent reasons cited. Mobilisation (sometimes early) was reported 65 times as the reason why prophylaxis was not prescribed. 'Early and aggressive mobilisation' was actually listed as a prophylactic measure in the study case report form. Fifteen patients in the private and 2 in the public sector were assessed as being at risk of bleeding according to the bleeding risk assessment (Table 3). There was an increased perception of bleeding risk in the private sector, probably reflecting the fact that a higher proportion of patients were on antiplatelet drugs such as aspirin and thienopyridines, e.g. clopidogrel. Notably, the surgeon scheduled to perform the procedure

Table 4. VTE risk assessment of patients before (clinically at risk) and after applying the Caprini RAM

\begin{tabular}{|c|c|c|c|c|c|c|}
\hline \multirow[b]{2}{*}{$\begin{array}{l}\text { Caprini VTE risk level } \\
\text { determined on TRFS }\end{array}$} & \multicolumn{2}{|c|}{ Private $(N=315)$} & \multicolumn{2}{|c|}{ Public $(N=138)$} & \multicolumn{2}{|c|}{ All $(N=453)$} \\
\hline & $\begin{array}{l}\text { Clinically at risk, } \\
n(\%)\end{array}$ & $\begin{array}{l}\text { RAM at risk, } \\
n(\%)\end{array}$ & $\begin{array}{l}\text { Clinically at risk, } \\
n(\%)\end{array}$ & $\begin{array}{l}\text { RAM at risk, } \\
n(\%)\end{array}$ & $\begin{array}{l}\text { Clinically at risk, } \\
n(\%)\end{array}$ & $\begin{array}{l}\text { RAM at risk, } \\
n(\%)\end{array}$ \\
\hline Low (TRFS 0 - 1) & $-(0)$ & $15(4.7)$ & $3(33.3)$ & $9(6.5)$ & $3(12.5)$ & $24(5.3)$ \\
\hline Moderate (TRFS 2) & $6(12.2)$ & 49 15.6) & $12(63.2)$ & $19(13.8)$ & $18(26.5)$ & $68(15.0)$ \\
\hline High (TRFS 3 - 4) & $46(39.0)$ & $118(37.5)$ & $42(93.3)$ & $45(32.6)$ & $88(54.0)$ & $163(36.0)$ \\
\hline Highest (TRFS $\geq 5$ ) & $97(72.9)$ & $133(42.2)$ & $63(96.9)$ & $65(47.1)$ & $160(80.1)$ & $198(43.7)$ \\
\hline Total & $149(47.3)$ & $315(100)$ & $120(87.0)$ & $138(100)$ & $269(59.4)$ & $453(100)$ \\
\hline
\end{tabular}


Table 5. VTE prophylaxis according to the Caprini RAM

\begin{tabular}{|c|c|c|c|c|c|c|}
\hline \multirow[b]{2}{*}{$\begin{array}{l}\text { Caprini VTE risk level } \\
\text { determined on TRFS }\end{array}$} & \multicolumn{2}{|c|}{$\begin{array}{l}\text { Private } \\
(N=315)\end{array}$} & \multicolumn{2}{|c|}{$\begin{array}{c}\text { Public } \\
(N=138)\end{array}$} & \multicolumn{2}{|c|}{$\begin{array}{c}\text { All } \\
(N=453)\end{array}$} \\
\hline & Patients, $n(\%)$ & $\begin{array}{l}\text { Prophylaxis } \\
\text { prescribed, } n(\%)^{*}\end{array}$ & Patients, $n(\%)$ & $\begin{array}{l}\text { Prophylaxis } \\
\text { prescribed, } n(\%)^{*}\end{array}$ & Patients, $n(\%)$ & $\begin{array}{l}\text { Prophylaxis } \\
\text { prescribed, } n(\%)^{*}\end{array}$ \\
\hline Low (TRFS 0 - 1) & $11(3.5)$ & $9(81.8)$ & $9(6.5 \%)$ & $2(22.2)$ & $20(2.4)$ & $11(55.0)$ \\
\hline Moderate (TRFS 2) & $42(13.3)$ & $40(95.2)$ & $19(13.8)$ & $15(78.9)$ & $61(13.5)$ & $55(90.2)$ \\
\hline High (TRFS 3 - 4) & $125(39.7)$ & $121(96.8)$ & $43(31.2)$ & $35(81.4)$ & $168(37.1)$ & $156(92.9)$ \\
\hline Highest (TRFS $\geq 5$ ) & $137(43.5)$ & $135(98.5)$ & $67(48.5)$ & $58(86.6)$ & $204(45.0)$ & $193(94.6)$ \\
\hline Total & $315(100)$ & $305(96.8)$ & $138(100)$ & $110(79.7)$ & $453(100)$ & 415 (91.6) \\
\hline
\end{tabular}

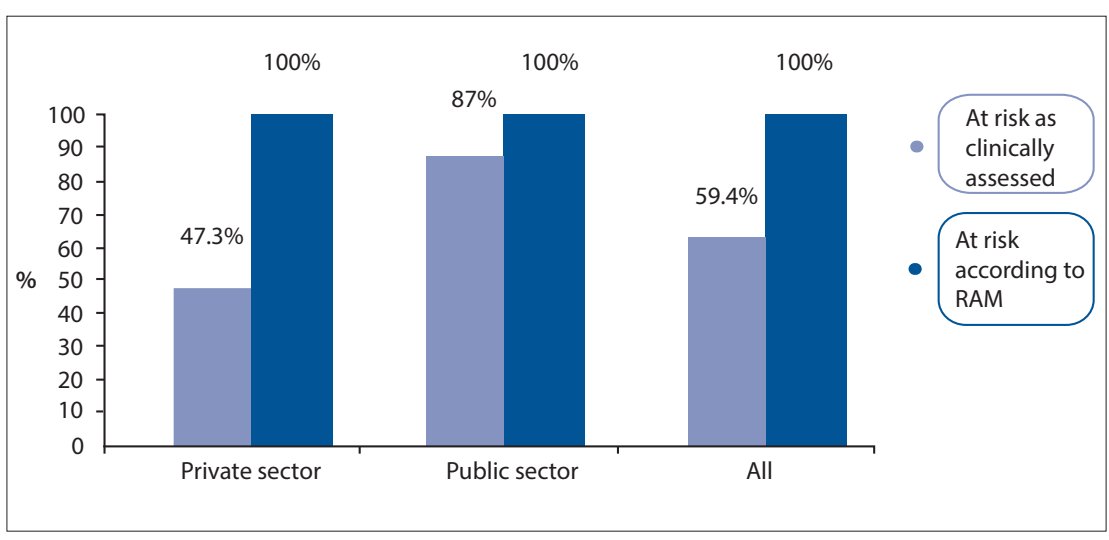

Fig. 1. VTE risk assessment: clinical v. RAM TRFS (VTE = venous thromboembolism; RAM = risk assessment model; TRFS = total risk factor score.)

was also the assessor of bleeding risk in the private sector. A bias therefore existed to prevent postoperative bleeding, and possible litigation, in the private sector. Other reasons for omitting prophylaxis included need for ventilation, surgery in a vulnerable site, e.g. the spine, and presence of a haematoma. More than one reason for the omission of VTE prophylaxis was reported for some patients.

\section{Prophylaxis prescribed}

The distribution of patients according to healthcare sector, Caprini risk score and VTE prophylaxis prescribed is set out in Table 5. Percentages were calculated out of the sample sizes for private $(n=315)$, public $(n=138)$ and all $(N=453)$ patients.

VTE prophylaxis was prescribed to 110 (79.7\%) of the 138 public sector patients, of whom $81.4 \%$ in the high-risk level and $86.6 \%$ in the highest-risk level received prophylaxis. VTE prophylaxis was prescribed to 305 of the 315 private patients $(96.8 \%)$, of whom $96.8 \%$ in the high-risk level and $98.5 \%$ in the highest-risk level received prophylaxis.

The chemoprophylaxis regimens differed between the private and public sectors, possibly owing to availability of preparations (reflecting state tenders in the public sector), drug cost (unfractionated heparin (UFH) costs less than low-molecular-weight heparin (LMWH)), and VTE prophylaxis protocols in specific medical institutions.

The use of LMWH predominated in the private sector, with $92 \%$ of patients receiving LMWH and the balance (8\%) oral factor Xa inhibitors. Low-dose UFH was not prescribed in the private sector. Conversely, only $67.5 \%$ of public sector patients received LMWH, the rest receiving UFH. There was no use of oral factor Xa inhibitors in the public sector. The LMWH dose was most commonly $40 \mathrm{mg}$ once twice daily. The use of non-chemotherapeutic prophylactic VTE prophylaxis was extremely limited in both the private and the public sectors, with pneumatic stockings being used in $<3 \%$ of patients.

\section{Duration of prophylaxis}

The overall duration of prophylaxis across all risk levels was 6.5 days and was similar in both healthcare sectors. The average duration of prophylaxis for high- and highest-risk patients, however, was 4.89 and 7.33 days in the private and public sectors, respectively. The longer duration of prophylaxis in patients in the high/ highest-risk groups in the public v. the private daily in both sectors and that of UFH 5000 IU sector probably relates to the longer duration of admission in the public sector.

\section{Conclusion}

The TUNE-IN Wave 2 registry provided valuable insight regarding the use of routine patient risk assessment in surgical inpatients in both the private and the public sectors. The registry determined that all the surgical inpatients in the study population were at risk of VTE to some extent. Doctors do not routinely formally risk-assess patients, and use of a formal RAM increases their awareness of patient risk. The results of this study mirror those of the TUNE-IN study, confirming the Caprini RAM to be applicable across all healthcare sectors in SA.

Before applying the Caprini RAM, the admitting doctors clinically diagnosed only 269 (59.4\%) of the patients to be at risk for VTE. Public sector patients were clinically assessed as being at risk for VTE more commonly than those in the private sector $(87.0 \%$ v. $47.3 \%)$. However, all the patients in the study were at risk to a greater or lesser extent as assessed on the RAM.

After applying the Caprini RAM, VTE prophylaxis was prescribed to 415 (91.6\%) of the 453 patients in the study. Prophylaxis was prescribed for $92.9 \%$ of the patients in the high-risk category and to $94.6 \%$ of those in the highest-risk category. A greater percentage of patients across all risk levels in the private $\mathrm{v}$. the public sector received prophylaxis $(96.8 \%$ v. $79.7 \%)$, possibly owing to availability of chemoprophylaxis drug preparations, costs and VTE prophylaxis protocols. Despite all the patients in the study being at risk to some extent, $8.4 \%$ did not receive any form of prophylaxis. Assessment of VTE risk as being low was the most frequent reason for not prescribing prophylaxis in both healthcare sectors.

Early and aggressive mobilisation continues to be seen as an active form of VTE prophylaxis in both sectors. It was the sole 
form of prophylaxis in $47.6 \%$ of the private and $20.3 \%$ of the public sector patients. Chemoprophylaxis prescribed included LMWH, lowdose UFH and oral factor Xa inhibitors, and was prescribed to $41.6 \%$ of the private and $58.7 \%$ of the public sector patients with or without concomitant use of graduated compression stockings or intermittent pneumatic compression.

The recommended doses of chemoprophylaxis in VTE prophylaxis guidelines appear to be being adhered to in both sectors. Forty milligrams of LMWH daily and 5000 IU UFH twice daily were the doses most commonly prescribed. The average duration of prophylaxis across all risk levels was similar in the two sectors (6.2 days in the private and 7.0 days in the public sector), with an overall average of 6.5 days.

There is room for improvement in both the public and the private sectors with respect to identifying and assessing patients at risk of VTE, as well as provision of correct prophylaxis. The implementation of a formalised VTE risk assessment tool will benefit patients and ensure standardisation of VTE risk assessment and administration of adequate prophylaxis.
Declarations and ethics approval. This study was funded by Sanofi South Africa (Pty) Ltd. Dr Wayne Riback is employed by Sanofi as Senior Medical Advisor. Data analysis was prepared and compiled by the Data Management Department of Sanofi, supported by an independent data report via a contracted third party. Ethical approval for the study was obtained from Pharma Ethics and the relevant ethics committees of the participating hospitals.

\section{References}

1. Cohen AT, Tapson VF, Bermann J-F, et al. Venous thromboembolism risk and prophylaxis in the acute hospital care setting (ENDORSE study): A multinational cross-sectional study. Lance 2008;371(9610):387-394. [http://dx.doi.org/10.1016/S0140-6736(08)60202-0]

2. Guyatt GH, Akl EA, Crowther M, et al. American College of Chest Physicians Antithrombotic Therap and Prevention of Thrombosis Panel. Antithrombotic therapy and prevention of thrombosis. 9th ed. Chest 2012;141(2 Suppl):7S-47S. [http://dx.doi.org/10.1378/chest.1412S3]

3. Jacobson BF, Louw S, Büller $\mathrm{H}$, et al. Venous thromboembolism - prophylaxis and therapeutic practice guidelines. S Afr Med J 2013;103(4):261-267. [http://dx.doi.org/10.7196/samj.6706

4. Wessels P, Riback WJ. DVT prophylaxis in relation to patient risk profiling - the TUNE-IN study. S Afr Med J 2012;102(2):85-89.

Accepted 9 September 2014. 